\title{
Optimal intensity targets for greenhouse gas emissions trading under uncertainty
}

\author{
Frank Jotzo • John C. V. Pezzey
}

Received: 22 June 2005 / Accepted: 14 December 2006 / Published online: 27 February 2007

(C) Springer Science+Business Media B.V. 2007

\begin{abstract}
Uncertainty is an obstacle for commitments under cap and trade schemes for emission permits. We assess how well intensity targets, where each country's permit allocation is indexed to its future realized GDP, can cope with uncertainties in international greenhouse emissions trading. We present some empirical foundations for intensity targets and derive a simple rule for the optimal degree of indexation to GDP. Using an 18-region simulation model of a cooperative, global cap-and-trade treaty in 2020 under multiple uncertainties and endogenous commitments, we show that optimal intensity targets could reduce the cost of uncertainty and achieve significant increases in global abatement. The optimal degree of indexation to GDP would vary greatly between countries, including super-indexation in some advanced countries, and partial indexation for most developing countries. Standard intensity targets (with one-to-one indexation) would also improve the overall outcome, but to a lesser degree and not in all individual cases. Although target indexation is no magic wand for a future global climate treaty, gains from reduced cost uncertainty and the potential for more stringent environmental commitments could justify the increased complexity and other potential downsides of intensity targets.
\end{abstract}

Keywords Climate policy · Emissions trading - Uncertainty · Intensity targets · Optimality $\cdot$ Simulation modelling

JEL categories Q Q540 - Q580 - D800

\section{F. Jotzo $(\bowtie)$}

Research School of Pacific and Asian Studies, The Australian National University, Canberra, ACT 0200, Australia

e-mail: frank.jotzo@anu.edu.au

J. C. V. Pezzey

Centre for Resource and Environmental Studies, The Australian National University, Canberra, ACT 0200, Australia

e-mail: pezzey@cres.anu.edu.au 


\section{Introduction}

Uncertainty can be a major impediment for cap-and-trade schemes for emission permits, be they greenhouse gases or other pollutants. Setting a fixed emission cap or target for each participant can give greater certainty about future emission levels and perhaps environmental impacts, but in so doing it creates uncertainties about compliance costs. Economic uncertainty is often a rallying point for opposition against environmental policy, whether implemented by regulation or by market mechanisms. Where compliance costs are uncertain, overall levels of environmental commitment tend to be watered down. Where those regulated have a strong degree of sovereignty, as in international negotiations, uncertainty can even preclude an agreement altogether.

International climate negotiations are a case in point. After the Kyoto Protocol was signed in 1997, debate raged over how much the Kyoto commitments would cost (Toman 2004). Estimates diverged widely (Weyant 1999), and the fact that meeting the Protocol's fixed quantity targets might have led to comparatively high and uncertain costs, even under international emissions trading, contributed to the US pulling out of the agreement. Bringing developing countries on board, essential for a meaningful post-Kyoto treaty, faces even greater challenges from economic uncertainty. Poor countries' decision-makers can ill afford to sign a treaty that risks major cost blow-outs or 'stifling development'. This matters, because although it is well established in theory that price control (i.e. a tax) is preferable to quantity control (i.e. cap-and-trade) under cost uncertainty and for pollutants with a flat marginal damage function, such as greenhouse gases (Weitzman's 1974; Pizer 2002), capand-trade is fast becoming the dominant instrument for national and international greenhouse gas control. The European $\mathrm{CO}_{2}$ Emissions Trading Scheme (ETS) is the largest commitment to this instrument so far, and a number of US States (Northeastern States and California) are planning or implementing their own cap-and-trade systems.

It is therefore of key relevance to climate policy to ask: can cap-and-trade schemes be better designed to reduce uncertainty about compliance costs? If so, environmental agreements can become both more achievable and more effective, by including targets which are more stringent overall, even if not at every place and time. Of several design features proposed to reduce uncertainty, perhaps the most widely discussed, and the one we study here, is making emission targets more flexible by indexing target allocations to GDP. They then become targets that include the emissions/GDP ratio, that is, emissions intensity targets (also referred to as 'relative', 'rate-based' or 'dynamic' targets), designed to compensate for fluctuations in emissions caused by fluctuations in economic activity.

Target indexation has been proposed as a way for making it easier for developing countries to commit to greenhouse targets, and featured in Argentina's greenhouse target proposed in the aftermath of the Kyoto negotiations. The Bush administration, after rejecting the Kyoto Protocol in 2001, set a target for future carbon intensity of the US economy. Even though this particular pledge is not binding and implies little effort, it sparked renewed interest in the concept of intensity targets. Canada also is looking to set its own greenhouse gas intensity targets. US support for intensity targets brought a greater political dimension to what is essentially a technical issue of mechanism design. Intensity targets have been criticised for increasing uncertainty 
in year-by-year emission levels under a treaty, possibly weakening environmental commitments, and for bringing greater complexity. ${ }^{1}$

Here we take a single-period, stochastic, globally integrated, though mainly partial equilibrium, theoretical model of emissions trading with flexible targets under uncertainty, named Mechanisms for Abating Total Emissions under Stochasticity (MATES), and apply it empirically to extend the analysis of greenhouse intensity targets under uncertainty significantly beyond that in the literature so far. ${ }^{2}$ Crucially, MATES is a multi-country model, which automatically transmits uncertainty between countries via the global permit price (though total abatement is determined by maximising global welfare rather than by strategic interactions among countries); and we use it in a way that allows expected target levels to be endogenous, that is, to fall (become more stringent) in response to the lower cost of uncertainty itself that intensity targets deliver for risk-averse countries. This is in contrast to existing analytic contributions by Sue Wing et al. (forthcoming), Quirion (2005) and Kolstad (2005), all of which considered single countries with fixed (exogenous) expected target levels.

MATES also allows general intensity targets, defined by a continuous degree of indexation to GDP, and thus allows a distinction between standard intensity targets, with one-to-one indexation, and optimal intensity targets, where a partial degree of indexation is individually chosen to minimise a country's cost of uncertainty. Partial indexation has been considered by Sue Wing et al. (forthcoming) and earlier in a foundation, less analytic paper (Ellerman and Sue Wing 2003), but here in a multi-country model it yields initially surprising recommendations for the degrees of indexation that different types of countries should use.

In Sect. 2 we discuss the empirical underpinnings of intensity targets, give the formal definition in MATES of general intensity targets under uncertainty, and derive our rule for optimal indexation. Section 3 describes the MATES permit trading model under uncertainty and risk aversion, and its calibration as an 18-country (or region) model of a cooperative (non-free-riding) post-Kyoto treaty for all countries and most greenhouse gas emissions in 2020. Simulation results and sensitivity analysis are presented in Sect. 4, for scenarios with absolute targets, standard intensity targets (with one-to-one indexation) and optimal intensity targets. Section 5 discusses issues of framing, potential drawbacks and practical applicability of intensity targets, and here we especially address the criticism that intensity targets increase emissions uncertainty and thereby undermine environmental integrity. Section 6 concludes.

1 On proposals for intensity targets as a way to draw developing countries into a climate agreement, see Baumert et al. (1999), Frankel (1999), Lutter (2000), Philibert and Pershing (2001); on the Argentine target, see Bouille and Girardin (2002), Barros and Conte Grand (2002); on the Bush 'target', see for example, van Vuuren et al. (2002), Blanchard and Perkaus (2004); and on criticism of target indexation, Dudek and Golub (2003), Müller and Müller-Fürstenberger (2003).

2 A companion paper, Pezzey and Jotzo (2007), defines the MATES model in more detail, including its coverage of an emissions tax and non-tradable emission targets, and derives theoretical results for expected net benefit under risk neutrality. 


\section{Formulation of intensity targets}

After discussing the empirical background for intensity targets, here we analyse future business-as-usual (BAU) emissions as a function of three separate uncertainties. ${ }^{3}$ This is complemented by a generalized formulation of emission targets indexed continuously to GDP. We then derive a simple rule for optimal indexation of targets, and show the conditions for a 'standard' intensity target with one-to-one indexation to reduce net emissions uncertainty compared to an absolute target.

\subsection{Empirical background}

The concept of intensity targets as a means to reduce uncertainty rests on the assumption that GDP and emissions move together. To this end, we are interested in the co-movement of fluctuations in emissions and fluctuations in GDP - what happens to emissions when the economy grows at below or above average rates. ${ }^{4}$

Several studies have examined the GDP-emissions relationship empirically in the context of intensity targets, using various methods including statistical forecasting models applied to historical data and analysis of past published emissions forecasts (Lutter 2000; Sue Wing et al. forthcoming; Philibert 2004), analysis of co-movement of series over time (Höhne and Harnisch 2002), and country case studies (Kim and Baumert 2002; Bouille and Girardin 2002). Each of these studies confirms that emissions tend to fluctuate to some degree with fluctuations in GDP, though the nature and degree of linkage estimated varies.

Our own empirical work (Jotzo 2006b) shows a significant positive correlation between deviations of GDP from trend and deviations of emissions from fossil fuel combustion from trend, for 23 of the 30 largest emitting countries over the period 1971-2000. The strength of the correlation varies greatly between countries, with a mean and median around one. Thus, in most countries, emissions on average move in tandem with GDP, but with large divergences from the mean in individual episodes due to changes in emissions intensity. No such correlation is evident between GDP and non- $\mathrm{CO}_{2}$ greenhouse gas emissions, and between GDP and emissions from land-use change.

Intensity targets will be able to deal only with uncertainty about future GDP, not uncertainty about future emissions intensity, or uncertainty in parts of the economy where emissions are independent of GDP. For the calibration of the simulation model described below, we need empirical estimates of the magnitude of these uncertainties. Results from statistical forecasting models applied to historical data (again see Jotzo 2006b) indicate that uncertainty about future GDP is sizeable, but significantly smaller than uncertainty about emissions intensity. Uncertainties are greater in

\footnotetext{
3 Throughout, we treat 'uncertainty' as the same, quantifiable concept as 'risk', rather than Knightian (unquantifiable) uncertainty.

4 This question is quite distinct from the long-term structural relationship between economic growth and greenhouse emissions that is the subject of most of the ample literature on the relationship between GDP and emissions, including the Environmental Kuznets Curve hypothesis (Holtz-Eakin and Selden 1995; Schmalensee et al. 1998; Galeotti et al. 2006; Dinda 2004); and the causal relationship between energy use and economic activity (e.g. Oh and Lee 2004; Asafu-Adjaye 2000).
} 
non-OECD than in OECD countries; and uncertainty about non-energy sector emissions is of a similar broad magnitude as that for energy-sector (fuel combustion) emissions intensity. We will assume that realizations of random variables are independent of each other, and in particular that deviations of emissions intensities in the energy sector from their expectations are independent of GDP deviations. (See below and footnote 5.)

\subsection{Future emissions and uncertainties}

Our application of MATES to global greenhouse gas abatement assumes that emissions in one part of the economy are linked with GDP, though the link is not perfect because emissions intensity also fluctuates. Aggregate uncertainty about future BAU emissions in the model then stems from three separate sources. They are:

1. uncertain output, measured by GDP and denoted $\widetilde{Y}_{i} \$ /$ year, where $i$ denotes one of the many countries or regions into which the world is divided (18 of them in our empirical model), and \$ means constant 2,000 US dollars;

2. uncertain emissions intensity of output in the 'linked' part of the economy, denoted $\tilde{\eta}_{i} \mathrm{t} / \$$, where $\mathrm{t}$ means a tonne of $\mathrm{CO}_{2}$-equivalent emissions; and

3. uncertainty in other, not GDP-linked emissions.

Future BAU emissions in a particular random realization are thus equal to expected BAU emissions times adjustments for expectation errors for GDP, emissions intensity and emissions in the non-linked sector.

Formally, realized BAU emissions for country $i$ are modelled as

$$
\widetilde{E}_{i}^{b}=E_{i}^{b}\left[1+\alpha_{i}\left(\varepsilon_{Y i}+\varepsilon_{\eta i}\right)+(1-\alpha) \varepsilon_{\rho i}\right]
$$

where our notation is

$\widetilde{E}_{i}^{b}=$ Actual BAU emissions in a particular random realization (in t/year)

$E_{i}^{b}=$ Expected BAU emissions

$\alpha_{i}=$ Fixed share of the economy where emissions are linked with GDP $(0 \leqslant$ $\left.\alpha_{i} \leqslant 1\right)$

$\varepsilon_{Y i}=$ Proportional deviation ('error') of actual GDP $\tilde{Y}_{i}$ from its expectation $Y_{i}$

$\varepsilon_{\eta i}=$ Proportional deviation of actual emissions intensity $\tilde{\eta}_{i}$ in the 'linked' sector from its expectation $\eta_{i}$

$\varepsilon_{\rho i}=$ Proportional deviation of actual emissions from expectations in the "nonlinked' sector.

A tilde $(\sim)$ superscript above and below denotes a particular realization of a random variable, whereas no superscript denotes the expectation of a random variable. The disappearance of subscript $i$ or $k$ below denotes summing over all countries: $\Sigma_{i} J_{i}=\Sigma_{k} J_{k}=: J$, for any variable or parameter $J$.

Error terms $\varepsilon_{Y i}$ and $\varepsilon_{\eta i}$ are assumed to be additive rather than multiplicative, to keep the stochastic analysis tractable. We assume that the error terms are distributed 
normally and are independent of each other, ${ }^{5}$ with ${ }^{6}$

$$
\varepsilon_{Y i} \sim N\left(0, \sigma_{Y i}\right), \varepsilon_{\eta i} \sim N\left(0, \sigma_{\eta i}\right) \quad \text { and } \quad \varepsilon_{\rho i} \sim N\left(0, \sigma_{\rho i}\right) .
$$

So GDP uncertainty $\varepsilon_{Y i}$ affects emissions in the $\alpha_{i}$ proportion of the economy, but structural shifts and other random influences $\left(\varepsilon_{\eta i}\right)$ also play a role here; while in the other, $\left(1-\alpha_{i}\right)$ proportion, emissions are independent of GDP and subject to random shocks $\varepsilon_{\rho i}$.

The $\sigma_{i}$ parameters are measures of the degree of uncertainty. Numerical calibration is done based on the empirical estimates described in Sect. 2.1 above (see also Appendix). Again following empirical findings, we assume that the share $\alpha_{i}$ of emissions linked with GDP in each region is equal to the share of the energy sector in total emissions. This assumption would obviously need to be refined for detailed country-level analyses, which ideally would rely on more disaggregated data.

Expected BAU emissions $E_{i}^{b}$, GDP $Y_{i}$ and population $L_{i}$ are calibrated on the basis of levels reported for the years 2002/2000, and forecasts by the main forecasting agencies. The Appendix gives sources and shows in Table 5 all parameter values used.

\subsection{Emissions targets}

Next, our greenhouse version of MATES defines a general flexible target (or permit allocation) as a proportion of expected future BAU emissions, indexed to realized GDP as follows:

$$
\tilde{X}_{i}=x_{i} E_{i}^{b}\left(1+\beta_{i} \varepsilon_{Y i}\right),
$$

with notation

$\tilde{X}_{i}$ realized target (in t/year)

$x_{i}$ target as a proportion ( $>0$, perhaps $>1$ ) of expected future BAU emissions (also known as 'target stringency' or 'target tightness' where 'more stringent' means a lower $x_{i}$ )

$\beta_{i}$ degree of indexation of the target to GDP $(\geqslant 0)$ and $E_{i}^{b}$ and $\varepsilon_{Y i}$ are as defined above.

By how much the realized target gets adjusted for a deviation in GDP from its expected value depends on $\beta_{i}$, its degree of indexation. Two obvious special cases are

Absolute target, with no indexation $\left(\beta_{i}=0\right)$, hence

$$
\tilde{X}_{i}=X_{i}=x_{i} E_{i}^{b}
$$

Standard intensity target, with one-to-one indexation $\left(\beta_{i}=1\right)$, hence

$$
\widetilde{X}_{i}=x_{i} E_{i}^{b}\left(1+\varepsilon_{Y i}\right)
$$

5 In practice, these deviations from expectations will of course not always be uncorrelated, but there is little empirical basis for assuming any particular correlation structure. For example, Webster and Cho (2006) found positive correlation between GDP historical growth rates in some country pairs, and negative correlation in others. However, our empirical analysis (Jotzo 2006b) does not indicate systematic correlations between $\varepsilon_{Y i}, \varepsilon_{\eta i}$ and $\varepsilon_{\rho i}$. Given the difficulty in establishing empirical estimates, correlation between different regions is usually dealt in modelling studies by assuming perfect correlation or perfect independence (Webster and Cho 2006).

6 In the numerical simulations, these error terms (as well as the error term $\varepsilon_{C i}$ introduced below) are truncated at two standard deviations above and below zero. This is done in order to exclude unrealistic realizations at the extreme tails of the probability distributions. 
So in our terminology, Kyoto Protocol targets are absolute; and what the literature usually refers to as just 'intensity targets' are here standard intensity targets. Partial indexation $\left(0<\beta_{i}<1\right)$ is possible and is discussed below. Importantly, with no GDP uncertainty, absolute and intensity targets are the same because they use the same $x_{i}$.

\subsection{Optimal intensity targets}

Since the error term $\varepsilon_{Y i}$ appears in both realised BAU emissions and intensity targets, target indexation changes the variability of the effort implied by the target (the difference between BAU emissions and the target):

$$
\widetilde{E}_{i}^{b}-\widetilde{X}_{i}=E_{i}^{b}-X_{i}+\widetilde{N}_{E i} \text {, hence } \widetilde{E}^{b}-\tilde{X}=E^{b}-X+\widetilde{N}_{E},
$$

where from [2.1] to [2.3], a country's net emissions uncertainty (net of any neutralizing effect of the index $\beta_{i}$ ) is

$$
\tilde{N}_{E i}:=\left[\left(\alpha_{i}-\beta_{i} x_{i}\right) \varepsilon_{Y i}+\alpha_{i} \varepsilon_{\eta i}+\left(1-\alpha_{i}\right) \varepsilon_{\rho i}\right] E_{i}^{b} .
$$

The expectation of squared net emissions uncertainty, from [2.7] and [2.2], is also important:

$$
D_{E i}:=E\left[\widetilde{N}_{E i}^{2}\right]=\left[\left(\alpha_{i}-\beta_{i} x_{i}\right)^{2} \sigma_{Y i}^{2}+\alpha_{i}^{2} \sigma_{\eta i}^{2}+\left(1-\alpha_{i}\right)^{2} \sigma_{\rho i}^{2}\right] E_{i^{2}}^{b} .
$$

Under the modelling assumptions set out in the next Section, particularly the assumptions that all countries take the global permit price as given, the expected global net benefit of the treaty is maximized when squared net emissions uncertainty $D_{E i}$ is minimized for all countries. ${ }^{7}$ This is achieved by setting all $\beta_{i}$ such that $\alpha_{i}-\beta_{i} x_{i}=0$, so that the:

Optimal (degree of) indexation of emissions targets to GDP is

$$
\beta_{i}^{*}=\alpha_{i} / x_{i} \text { for all } i \text {. }
$$

Together with (2.3), this defines an

$$
\text { Optimal intensity target as } \tilde{X}_{i}=x_{i} E_{i}^{b}\left[1+\left(\alpha_{i} / x_{i}\right) \varepsilon_{Y i}\right]
$$

For some countries, the share of emissions linked with GDP, $\alpha_{i}$, may be greater than the target stringency expressed as a share of BAU emissions, $x_{i}$. That is, an optimal intensity target may be super-indexed to GDP $\left(\beta_{i}^{*}>1\right)$, a possibility which will be realized for several countries in our empirical analysis.

Our finding that not just the degree of GDP-emissions linkage, but also target stringency matters for the optimal degree of indexation, is in contrast to most earlier analyses on intensity targets which looked only at the emissions-GDP correlation, though it tallies with recent work by other authors. In a rather different analytical framework, Sue Wing et al. (forthcoming) found that to reduce variability in abatement burden in an economy with steady economic growth, “... stringent emission targets should be implemented using intensity limits, while lax targets should employ absolute limits".

7 This is shown formally in Pezzey and Jotzo (2007). We thank a referee for pointing out that under different market assumptions or net benefit functions, net benefit might not be maximized by minimizing all $D_{E i}$ 's. 
2.5 Does GDP indexation reduce uncertainty?

From (2.8), target indexation reduces uncertainty $D_{E i}$ if

$$
\left(\alpha_{i}-\beta_{i} x_{i}\right)^{2}<\alpha_{i}^{2}
$$

So from (2.9), optimal intensity targets are always expected to reduce uncertainty (unless $\alpha_{i}=0$ when optimal intensity and absolute targets coincide). The impact of standard intensity targets by contrast is ambiguous, because they undercompensate for GDP-related fluctuations in emissions in cases where $\beta_{i}^{*}>1$, and overcompensate where $\beta_{i}^{*}<1$. However, (2.11) holds whenever

$$
(0<) \beta_{i} x_{i}<2 \alpha_{i} \text {, which if } \beta_{i}=1 \text { is }(0<) x_{i}<2 \alpha_{i},
$$

so standard intensity targets $\left(\beta_{i}=1\right)$ are expected to reduce uncertainty unless $\alpha_{i}$, the degree of GDP-emissions linkage, is very small compared to $x_{i}$, the target stringency.

\section{Emissions trading under uncertainty}

We explain here how the multi-country, stochastic, mainly partial equilibrium MATES model handles emissions trading under uncertainty. To simulate the performance of intensity targets, we apply the model to the case of a global, post-Kyoto climate treaty, covering all greenhouse gases and taking effect in a single future time period, chosen here as the year 2020. We have chosen to divide the world into 18 regions or countries, hereafter just 'countries'. Five are high-income countries known together as 'the North', while 13 are low-income ones or 'the South'. Our choice both represents the main players in global climate policy as single countries (such as the USA, EU, China and India), and allows detailed analysis for selected developing countries like Argentina and South Korea. The empirical calibration reflects key features of individual countries, while not aiming at 'exact' numerical representation. For a list of the 18 countries and their empirical calibration, see Table 5 in the Appendix.

\subsection{Abatement costs and benefits, and permit trading}

The abatement treaty grants a flexible permit allocation $\left\{\widetilde{X}_{i}\right\}$, comprising an $\left(X_{i}, \beta_{i}\right)$ pair for each country which defines their $\widetilde{X}_{i}$ as in (2.3). We assume that the treaty allows permit trading, and assume perfect enforcement in the global permit market, so abated emissions equal the target only globally:

$$
\widetilde{E}=\widetilde{X} \text {, hence global abatement } \widetilde{Q}=\widetilde{E}^{b}-\widetilde{X} \text {. }
$$

The market price of a permit is $\tilde{p}$, assumed to be taken as given by all countries, and a country's emissions trading revenue is then

$$
\widetilde{R}_{i}:=\tilde{p}\left[\widetilde{X}_{i}-\widetilde{E}_{i}^{b}+\widetilde{Q}_{i}(\tilde{p})\right] \text {, with global } \widetilde{R} \equiv 0 \text { automatically. }
$$

8 Note that permit trading between countries could of course also occur outside of an international treaty (Rehdanz and Tol 2005). 
Country $i$ 's net benefit from emissions trading compared to no abatement anywhere ${ }^{9}$ is defined as ${ }^{10}$

$$
\begin{gathered}
\widetilde{A}_{i}:=\widetilde{B}_{i}-\widetilde{C}_{i} \$ / \text { year, where } \\
\widetilde{B}_{i}(\widetilde{Q}):=V_{i} \widetilde{Q}-1 / 2 W_{i}(\widetilde{Q})^{2}+\widetilde{R}_{i}, V_{i} \$ / t>0, W_{i} \$ \cdot \text { year } / t^{2}>0,
\end{gathered}
$$

is $i$ 's dollar-valued (gross) benefit $\widetilde{B}_{i}$ of global abatement $\widetilde{Q}$, including its emissions trading revenue. We include valuation of (or benefits from) global abatement in order to be able to determine the optimal level of abatement, in the broad vein of Nordhaus (1991). Abatement benefits allow us to model how the reduced uncertainty cost from using a potentially better type of target mechanism (such as intensity targets) would be spent endogenously on achieving lower target levels, which in turn means greater welfare. This "endogenous targets" effect is a key contribution here, and we discuss it, and the calibration of the benefit parameters $\left\{V_{i}\right\}$ and $\left\{W_{i}\right\}$, further in Sects. 3.3 and 3.4 , respectively.

The cost of $i$ 's own abatement $\widetilde{Q}_{i}$ is defined as

$$
\begin{aligned}
\widetilde{C}_{i}\left(\widetilde{Q}_{i}\right) & :=1 / 2 \widetilde{Q}_{i}^{2} / M_{i}+\widetilde{Q}_{i} \varepsilon_{C i}, M_{i} t^{2} / \$ . \text { year }>0 \\
& =1 / 2 \widetilde{Q}_{i}^{2} / M_{i}+\widetilde{Q}_{i} \widetilde{N}_{C i} / M_{i}, \text { with } \widetilde{N}_{C i}:=M_{i} \varepsilon_{C i} .
\end{aligned}
$$

The marginal abatement cost (MAC) curve is then linear in $\widetilde{Q}_{i}$, and uncertain:

$$
\widetilde{C}_{i}^{\prime}\left(\widetilde{Q}_{i}\right)=\widetilde{Q}_{i} / M_{i}+\varepsilon_{C i}=\widetilde{Q}_{i} / M_{i}+\widetilde{N}_{C i} / M_{i} .
$$

Here $\varepsilon_{C i}$ is Weitzman's (1974) 'pure unbiased [stochastic] shift' in the MAC. We assume $\mathrm{E}\left[\varepsilon_{C i}\right]=0, \mathrm{E}\left[\varepsilon_{C i}^{2}\right]=: \sigma_{C i}^{2}$, and $\varepsilon_{C i}$ is independent of all other uncertainties. ${ }^{11}$ Hence from [3.3], [3.4] and [3.5], realized net benefit is

$$
\widetilde{A}_{i}=V_{i} \widetilde{Q}-1 / 2 W_{i}(\widetilde{Q})^{2}-1 / 2 \widetilde{Q}_{i}^{2} / M_{i}-\widetilde{Q}_{i} \widetilde{N}_{C i} / M_{i}+\widetilde{R}_{i}
$$

A similar formula applies to the Unilateral case, denoted by the superscript $U$. Under unilateralism, each country decides on its own abatement effort $\widetilde{Q}_{i}^{U}$ in isolation (i.e. assuming $\partial \widetilde{Q}^{U} / \partial \widetilde{Q}_{i}^{U}=1$ ) and there is no trading revenue:

$$
\widetilde{A}_{i}^{U}:=V_{i} \widetilde{Q}^{U}-1 / 2 W_{i}\left(\widetilde{Q}^{U}\right)^{2}-1 / 2\left(\widetilde{Q}_{i}^{U}\right)^{2} / M_{i}-\widetilde{Q}_{i}^{U} \widetilde{N}_{C i} / M_{i}
$$

We do not model uncertainties in the benefits from global abatement, as under our independence assumptions, they would not affect the comparison of expected net benefit across mechanisms (though could perhaps have a minor effect on expected payoff, defined below). Stavins (1996), using a neglected result in Weitzman, noted

9 By comparing emissions trading with no abatement anywhere, rather than with no abatement by $i$ while all other countries abate according to the treaty, we are setting aside the problem of free riding; see also Sect. 3.3 below.

10 In a second-best world, this simple net benefit formula should be amended to allow for the marginal cost of public funds being greater than unity (Quirion 2004). Like several other features such as information and enforcement costs, this remains for further work, but it will in any case have little effect on the relative performance of absolute, standard intensity and optimal intensity targets as reported here. (see Pezzey and Jotzo 2007 for further details).

11 In practice, deviations in abatement costs from their expectations may well be correlated to a degree with deviations from expected emissions intensity. Nevertheless, a large share of MAC uncertainty would stem from not knowing in advance the aggregate responsiveness of greenhouse gas emitters to price signals, independent of future emissions levels and intensity. 
that this convenient result does not hold if benefit and cost uncertainties are correlated. However, there is no evidence for such correlation in the greenhouse case, or reason to suspect its existence.

The 'abatement potentials' $\left\{M_{i}\right\}$ in [3.5] are calibrated with reference to other studies, and the degree of uncertainty about abatement costs $\left\{\sigma_{C i}\right\}$ can be gleaned by comparing MAC estimates from different models, indicating substantial uncertainty about abatement opportunities and costs (see Appendix).

To maximize its financial benefit from emissions trading, each country chooses abatement $\widetilde{Q}_{i}$ to equate its MAC $\widetilde{C}_{i}^{\prime}$ in (3.6) to the permit price $\tilde{p}$, which gives

$$
\begin{aligned}
\widetilde{Q}_{i} & =\tilde{p} M_{i}-\widetilde{N}_{C i}, \text { and hence from (3.1) and (2.6) } \\
\tilde{p} & =\left(E^{b}-X+\widetilde{N}_{E}+\widetilde{N}_{C}\right) / M, \text { with expectation } \\
p & =\left(E^{b}-X\right) / M .
\end{aligned}
$$

Equation (3.10) clarifies an important reason for choosing a multi-country model. It is not just each country's own uncertainty that affects its position under emissions trading, but uncertainty in all other participating countries as well, transmitted through the realized global permit price $\tilde{p}$. How much this deviates from its expectation $p$ of course depends on deviations from expectations $\widetilde{N}_{E}$ in BAU emissions and $\widetilde{N}_{C}$ in MACs in all countries. So a flexible target that neutralizes some uncertainty in one country has flow-through effects for all others in the permit market.

\subsection{Payoff and risk aversion}

We assume here that country $i$ assesses the desirability of a move from unilateral to treaty abatement by calculating its expected payoff from the move, which is best viewed as comprizing three steps.

First, $i$ 's realized gain from the move is defined from (3.7) to (3.8) as the difference in realized net benefits between the treaty and unilateral outcomes:

$$
\widetilde{G}_{i}:=\widetilde{A}_{i}-\widetilde{A}_{i}^{U}
$$

Second, its realized payoff from the move is a risk-adjusted, strictly concave function of realized gain:

$$
\widetilde{U}_{i}:=\widetilde{G}_{i}+z_{i}\left(1-e^{-r \widetilde{G}_{i}}\right) \$ / \text { year; } \quad z_{i} \$ / \text { year }>0, r \text { year } / \$>0 .
$$

This captures $i$ 's aversion to risk, by weighting potential losses more heavily than potential gains, in line with what we perceive to be the political psychology of international treaties. Risk aversion is a prerequisite for modelling endogenous targets. Without risk aversion, the overall target $X$ is almost completely independent of the type of mechanism, and almost the only advantage of intensity targets is in reaching a given target at lower expected cost. ${ }^{12}$

\footnotetext{
12 With absolute and optimal intensity targets, the $\left(\alpha_{i}-\beta_{i} x_{i}\right)$ terms in (2.8) all become $\alpha_{i}$ or 0 ; and this means that without risk aversion (where $r=0$ so $U=G$ ), our maximizing criterion (3.15) below results in the same expected global target, $X$. With standard intensity targets, the $\left(\alpha_{i}-\beta_{i} x_{i}\right)$ terms are all $\alpha_{i}-x_{i}$; but for our calibration at least, this means that maximizing $G$ gives an $X$ only very slightly (well under 1\%) different from the $X$ found for absolute and optimal intensity targets.
} 
Finally, country $i$ 's expected payoff from the move (our measure of its rise in welfare) is then, since all the errors have normal distributions with zero means, ${ }^{13}$

$$
\begin{aligned}
U_{i}:= & \int_{R^{4 n}}\left[\widetilde{G}_{i}(\varepsilon)+z_{i}\left(1-e^{-r \widetilde{G}_{i}(\varepsilon)}\right)\right]\left[e^{-1 / 2 \Sigma\left[\left(\varepsilon_{Y i} / \sigma_{Y i}\right)^{2}+\left(\varepsilon_{\eta i} / \sigma_{\eta i}\right)^{2}+\left(\varepsilon_{\rho i} / \sigma_{\rho i}\right)^{2}+\left(\varepsilon_{C i} / \sigma_{C i}\right)^{2}\right]} /\right. \\
& \left.(2 \pi)^{2 n} \Pi\left(\sigma_{Y i} \sigma_{\eta i} \sigma_{\rho i} \sigma_{C i}\right)\right] \mathrm{d} \varepsilon, \text { with } \\
\varepsilon:= & \left(\varepsilon_{Y i}, \ldots \varepsilon_{Y n}, \varepsilon_{\eta i}, \ldots \varepsilon_{\eta n}, \varepsilon_{\rho i}, \ldots \varepsilon_{\rho n}, \varepsilon_{C i}, \ldots \varepsilon_{C n}\right) .
\end{aligned}
$$

This will be less than expected gain $G_{i}$, by virtue of the positive parameters $z_{i}$ and $r$. Importantly, the characterization in (3.12-3.14) assumes that the payoff a country perceives is framed solely in terms of the financial and environmental consequences of the treaty, not the economy overall. We discuss this framing effect further in Sect. 5.1.

Expectation results, including expected payoff, are computed numerically by Monte Carlo simulations in a multi-stage algorithm. The model is calculated for a large number of random realizations (here 10,000), with joint draws for each of the $18 \times 4=72$ stochastic parameters, and expectations computed as means over these realisations. In each simulation run, these calculations are done for a given target vector $\left\{x_{i}\right\}$. In iterative re-runs of the simulation, the distribution of targets $\left\{x_{i}\right\}$ between countries is then adjusted to fulfill an equity constraint which we will discuss next. Finally the overall target $X$ is adjusted to maximize global expected payoff $U$, again finding targets $\left\{x_{i}\right\}$ that fulfill the equity constraint. In simulations with optimal intensity targets, we jointly optimize targets $\left\{x_{i}\right\}$ and indexation $\left\{\beta_{i}\right\}$.

\subsection{Optimality, the equity criterion and endogenous targets}

For the case of a global climate treaty analysed here, we solve the model numerically as a cooperative game, by selecting both the global size $X$ and stringency distribution $\left\{x_{i}\right\}$ of expected targets, so that

1. expected global payoff $U$ is maximized as just noted, subject to:

2. an equity criterion that all countries have the same expected payoff per person from the Treaty: $U_{i} / L_{i}=U_{k} / L_{k}$ for all $i, k$, where $L_{i}, L_{k}$ are countries' populations

The equity constraint (3.16) is met solely through adjusting target stringencies $\left\{x_{i}\right\}$, which we feel is politically more realistic than cash transfers, as assumed for example by Bohm and Carlén (2002). Some constraint like (3.16) is needed to achieve a plausible differentiation of targets $\left\{x_{i}\right\}$ between countries under global payoff maximization with endogenous targets. With unconstrained maximization, countries that are least affected by uncertainty and risk aversion would be allocated very tight targets (low $x_{i}$ 's), while highly risk-averse countries would get implausibly generous targets. The equity criterion (3.16) is necessarily arbitrary (just as any exogenously imposed scheme of target differentiation would be), but in sensitivity analysis in Sect. 4.5, the choice of equity criterion turns out to make little difference to the relative merits of absolute and intensity targets. What we will call the Reference Case, to which results for all other scenarios will be compared, is the outcome of applying (3.15) and (3.16) to emissions trading with absolute targets, and results for this are given in Sect. 4.1 below.

13 The basic MATES model in Pezzey and Jotzo (2007) assumes risk-neutrality, and hence that countries maximize expected gain, not expected payoff. 
As foreshadowed above, a target type that neutralizes some of the uncertainty will give greater payoffs, and because criterion (3.16) does not specify a direct rule for target distribution, in turn this leads to tighter targets (a lower $X$ ), i.e. endogenous targets. We thus model how better mechanism design improves environmental outcomes from a treaty. Of the many proposed rules for, and subsequent analyses of, target setting or 'burden sharing' in greenhouse gas control (see for example Rose et al. (1998) or Berk and den Elzen 2001), most give exogenous targets.

As a participation constraint, we demand only that each country's payoff under the treaty is greater than if there was no treaty at all $\left(U_{i}>0\right)$. This quite weak constraint implicitly assumes that political cooperation can prevent free-riding (Eckersley 2004). The MATES model could be extended to non-cooperative situations and thus analyse free-riding incentives, but this is left for further work.

\subsection{Calibration of benefit and risk aversion parameters}

As already noted, endogenous target levels are a key policy feature of our model, and emerge from choosing target levels to maximize the net benefits of a policy mechanism, rather than just choosing a mechanism to minimize the cost of achieving exogenously fixed levels. But how did we calibrate the benefit valuation parameters $\left\{V_{i}\right\}$ and $\left\{W_{i}\right\}$ shown in Table 5? We chose not to use damage estimates as a proxy for each country's valuation of global abatement for three reasons: (a) damage estimates at the national level are highly speculative; (b) small differences in social discount rates translate to large differences in valuation because of the long time frames involved; and (c) high expected long-term damages from climate change in poorer countries do not necessarily translate to a correspondingly high willingness to pay for emission reductions now.

Instead, we calibrated the valuation parameters $\left\{V_{i}\right\}$ to conform broadly with preferences revealed by the international debate about burden sharing, and this calibration is illustrative in nature. Relative per capita valuations $\left\{V_{i} / L_{i}\right\}$ are assumed to be a function of per capita income and historical responsibility for greenhouse emissions (cumulative emissions of energy $\mathrm{CO}_{2}$ from 1970 to 2000 per capita), with per capita income weighted twice as heavily. This yields much higher $\left\{V_{i} / L_{i}\right\}$ in rich countries, but roughly an even split in total valuation $V$ between North and South. All other things being equal, this results in a greater relative abatement burden in richer countries, in line with the principle of common but differentiated commitments under the UN Framework Convention on Climate Change.

$W_{i}$, the slope of the marginal benefit of greenhouse gas abatement curve, is chosen to be a small constant times $V_{i}$, resulting in a slight upward slope of the marginal benefit curve, in line with the well-recognized notion that since most greenhouse gases are long-lived stock pollutants, the marginal damage curve is almost flat (Pizer 2002).

In calibrating the risk aversion parameters $\left\{z_{i}\right\}$ and $r$ in (3.13), we first choose all $z_{i}=1 / y_{i}$, with $y_{i}=Y_{i} / L_{i}$, per capita GDP. The $1 / y_{i}$ factor matches the stylized fact that uncertainty matters more in poor countries, and thus captures an essential feature of the global climate policy debate. The payoff concavity parameter $r$ is then calibrated so that risk aversion results in significantly less stringent commitments under uncertainty, but without stifling agreement altogether. In the default calibration, $r$ is chosen so that global abatement in the Reference Case is one quarter lower than it would be without risk aversion. Overall global valuation parameters $V$ and $W$, in conjunction with $r$, are chosen so that our Reference Case of a global climate treaty

Springer 
results in approximately a halving of global emissions growth between 2002 and 2020, compared to BAU.

\section{Performance of intensity targets}

How large are the potential improvements from intensity targets, and how important is optimal indexation? What are the key factors influencing the performance of intensity targets? This section explores these questions empirically through simulations using our model. We describe the Reference Case of our climate treaty with emissions trading, discuss aggregate and country-level results for the comparative performance of intensity targets, and then present alternative scenarios and sensitivity analysis.

\subsection{Reference Case}

The Reference Case assumes that all countries participate in a cap-and-trade treaty with absolute targets (no indexation, all $\beta_{i}=0$ ), covering all major greenhouse gases. The Reference Case is just a point of comparison for alternative scenarios with different types of intensity targets, not a prediction of a future climate treaty. Table 1 shows a summary of Reference Case results for the North/South country groups. Country targets $\left\{x_{i}\right\}$ are shown in Table 3 below.

Global abated emissions in the Reference Case, equal to the total amount of permits $(E=X)$, grow by $15 \%$ from 2002 to 2020 , compared to a projected $30 \%$ under BAU $\left(E^{b}\right)$. This is equivalent to a reduction of $12 \%$ below global BAU emissions $(x=0.88)$, as shown in Table 1 . The Reference Case thus describes a significant but arguably realistic level of effort. The expected amount of abatement undertaken under the treaty is $6.5 \mathrm{Gt} /$ year. By comparison, in the Unilateral case (no trading, no cooperation), expected abatement is only $0.9 \mathrm{Gt} / \mathrm{year}$, calculated using (3.8), and with marginal costs varying between countries. ${ }^{14}$

The expected permit price $p$ in the Reference Case from (3.11), equal to expected the marginal cost of abatement in all countries, is $15 \$ / t$ (of $\mathrm{CO}_{2}$-equivalent). A range of studies estimate discounted future marginal damage from climate change below this value, others higher. ${ }^{15}$ The permit prices in our modelling are comparable to prices paid for permits under the $\mathrm{EU} \mathrm{CO}_{2}$ Emissions Trading Scheme. Global dollar-valued global gain $G$ from the climate treaty, defined as the expected sum of (3.12), is over 50 billion $\$$ year, translating into a somewhat lower payoff $U$ after adjustment for risk aversion (from (3.14)). The expected global cost of abatement, $C$ from summing (3.5), is well below $0.1 \%$ of projected global GDP, which reassures us that this is an acceptable application of a partial equilibrium model.

The emission targets $\left\{x_{i}\right\}$ used for the Reference Case are chosen to satisfy (3.15) and (3.16), that is to maximize expected global (risk-adjusted) payoff from a treaty subject to per capita payoff being equalized across all countries. Targets are strongly differentiated between countries, with tighter targets applying where relative valuation

\footnotetext{
14 The magnitude of abatement in the Unilateral case, where each region maximizes their own expected benefit without regard to decisions by others, depends on the degree of aggregation. In a regionally more aggregated model, unilateral abatement would be larger, because each region captures a greater share of external (global) benefits of its abatement action.

$15 \mathrm{Tol}(2005)$ argued that the marginal damage costs of $\mathrm{CO}_{2}$ emissions are unlikely to exceed $\$ 50 / \mathrm{tC}$ or around $\$ 13.6 / \mathrm{tCO}_{2}$.
} 
Table 1 Results for Reference Case of treaty with emissions trading

\begin{tabular}{llcrc}
\hline Expected values of: & & Global & North & South \\
\hline Target (permits) as share of BAU emissions & $X_{i} / E_{i}^{b}=x_{i}$ & 0.88 & 0.79 & 0.93 \\
Permit price (\$/t) & $p$ & & 15.00 & \\
Expected reduction commitment (Gt/year) & $E_{i}^{b}-X_{i}$ & 6.46 & 3.89 & 2.57 \\
Expected abatement (Gt/year) & $Q_{i}$ & 6.46 & 1.39 & 5.07 \\
Expected permit exports (Gt/year) & $Q_{i}-\left(E_{i}^{b}-X_{i}\right)$ & 0 & -2.50 & 2.50 \\
Expected gain (cf. Unilateral, net of costs) (G\$/year) & $G_{i}$ & 56.5 & 7.2 & 49.4 \\
Expected (risk-adjusted) payoff (G\$/year) & $U_{i}$ & 47.6 & 6.8 & 40.8 \\
Expected costs from abatement (G\$/year) & $C_{i}$ & 57.3 & 12.3 & 44.9 \\
\hline
\end{tabular}

of abatement is higher, abatement is cheaper and uncertainty or risk aversion lower. Table 1 shows that on the whole, high-income 'Northern' countries have relatively stringent targets and buy permits, while poorer countries abate their emissions below their targets and sell the freed-up permits, taking advantage of their relatively generous targets and relatively greater abatement options (for more detail, see left hand columns in Table 3 below). Compared to base year (2002) emissions, 'Southern' countries' targets are above their current emissions, leaving room for future emissions growth. The South as a group would receive substantial permit revenue, almost offsetting their overall abatement cost.

\subsection{Exogenous intensity targets}

We report on two types of intensity targets:

1. standard intensity targets with one-to-one indexation to GDP for all countries $i$ $\left(\beta_{i}=1\right.$ as in [2.5]); and

2. optimal intensity targets, where $\beta_{i}^{*}=\alpha_{i} / x_{i}$ for all $i$ as in (2.10), so uncertainty due to fluctuations in GDP is fully neutralized.

In a first set of scenarios, we hold expected targets $\left\{X_{i}\right\}$ exogenously fixed at Reference Case levels, but index realized targets to GDP by setting all $\beta_{i}=1$ or $\alpha_{i} / x_{i}$. This shows just the cost effectiveness of using indexation to achieve a given global abatement target under uncertainty.

Global results are reported in Table 2. Expected abatement is unchanged by definition. The expected global gain $G$ increases by 5 and $7 \%$, respectively, under standard and optimal intensity targets. This estimated improvement in expected gain under fixed target levels does not depend on our assumptions about risk aversion and payoff, but derives directly from reduced cost uncertainty. Increases in (risk-adjusted) expected payoff $U$ from target indexation are substantially larger than in gain $G$, as they also take account of 'psychological' effects of a reduced risk of incurring losses under emissions trading.

Fixed, standard intensity targets do not necessarily increase in expected welfare in all countries. In some regions overcompensation under one-to-one indexation would in fact increase effective uncertainty relative to the Reference Case, as discussed in Sect. 4.4 below. 


\subsection{Endogenous intensity targets}

We now allow the set of countries' target levels, $\left\{x_{i}\right\}$, to be determined endogenously by maximizing global expected payoff subject to equal per capita distribution of expected payoffs, as in (3.6). With intensity targets neutralizing some or all of the GDP-related uncertainty, greater payoff can be achieved at a different set of maximizing target levels to those under absolute targets. Our results show the degree to which this reduced uncertainty can achieve both tougher environmental commitments, i.e. lower $X=\Sigma x_{i} E_{i}^{b}$, hence higher (expected global) abatement, $Q=E^{b}-X$, and higher (expected global) payoff $U$. With endogenous intensity targets, abatement increases by around one quarter (Table 2, last two columns). Under optimal indexation, slightly larger improvements are possible than with standard intensity targets, because not just target levels $\left\{x_{i}\right\}$ but also indexation levels $\left\{\beta_{i}^{*}\right\}$ are simultaneously free to vary (using (2.9) when maximizing $U$ in (3.15)). By contrast, the one-to-one indexation of standard intensity targets will on average over- or under-compensate for fluctuations in GDP. These quantitative estimates should be seen in the context of maximum improvement achievable if there was no uncertainty, which is one-third, deriving from our above calibration of risk aversion parameter $r$ (again see Table 2).

Both gain and payoff are significantly greater under endogenous intensity targets (14-37\% improvements over the Reference Case of absolute targets), compared to the corresponding scenarios with fixed, exogenous intensity targets (5-27\% improvements). This is because in addition to part of their uncertainty being neutralized, countries are free to choose their levels of abatement (and then generally choose higher levels, closer to what would happen with no uncertainty). These results would hold also if not all countries take on intensity targets. Reduced uncertainty in one part of the world is transmitted between countries through less variability in the permit price, and in our modelling context, through greater overall abatement and payoff sharing.

Table 2 Intensity targets and emissions trading: global results

\begin{tabular}{|c|c|c|c|c|c|c|}
\hline & \multirow{2}{*}{$\begin{array}{l}\text { Certainty } \\
\text { equivalence } \\
\left(\text { all } \sigma_{i}=0\right)\end{array}$} & \multirow{2}{*}{$\begin{array}{l}\text { Absolute } \\
\text { targets } \\
\text { (Reference } \\
\text { Case, all } \\
\left.\beta_{i}=0\right)\end{array}$} & \multicolumn{2}{|c|}{$\begin{array}{l}\text { Fixed exogenous targets } \\
\text { (all } x_{i} \text { as in Reference Case) }\end{array}$} & \multicolumn{2}{|c|}{$\begin{array}{l}\text { Endogenous targets } \\
\text { (all } x_{i} \text { optimized) }\end{array}$} \\
\hline & & & $\begin{array}{l}\text { Standard } \\
\text { intensity } \\
\text { targets (all } \\
\left.\beta_{i}=1\right)\end{array}$ & $\begin{array}{l}\text { Optimal } \\
\text { intensity } \\
\text { targets (all } \\
\left.\beta_{i}^{*}=\alpha_{i} / \mathrm{x}_{i}\right)\end{array}$ & $\begin{array}{l}\text { Standard } \\
\text { intensity } \\
\text { targets (all } \\
\beta_{i}=1 \text { ) }\end{array}$ & $\begin{array}{l}\text { Optimal } \\
\text { intensity } \\
\text { targets (all } \\
\left.\beta_{i}^{*}=\alpha_{i} / x_{i}\right)\end{array}$ \\
\hline \multicolumn{7}{|c|}{ Expected values of: } \\
\hline $\begin{array}{l}\text { Abatement } Q \\
\text { (Gt/year) }\end{array}$ & 8.6 & 6.5 & 6.5 & 6.5 & 8.0 & 8.2 \\
\hline $\begin{array}{l}\text { Gain } G \\
\text { (billion } \$ / \text { year) }\end{array}$ & 75.9 & 56.5 & 59.4 & 60.7 & 64.7 & 66.3 \\
\hline $\begin{array}{l}\text { Payoff } U \\
\text { (billion } \$ / \text { year) }\end{array}$ & 76.6 & 47.6 & 58.5 & 60.7 & 62.4 & 65.2 \\
\hline \multicolumn{7}{|c|}{ Percentage difference compared to absolute targets: } \\
\hline Abatement $Q(\%$ & 33 & - & 0 & 0 & 24 & 27 \\
\hline Gain $G(\%)$ & 34 & - & 5 & 7 & 14 & 17 \\
\hline Payoff $U(\%)$ & 61 & - & 23 & 27 & 31 & 37 \\
\hline
\end{tabular}


Nevertheless, it remains true that in this multi-region model, gains from cooperation and trade itself are significantly greater than gains from target indexation and endogeneity. The Reference Case's gain in net benefit compared to Unilateral action (as in (3.12)), and therefore due just to cooperation and trade, is $\$ 56.5 \mathrm{bn} / \mathrm{year}$. Moving further to our best target type, optimal intensity targets with endogenously chosen target levels, adds only another $\$ 9.8 \mathrm{bn} /$ year. However, allowing for risk aversion by considering payoff changes this decomposition significantly: $\$ 47.6 \mathrm{bn} / \mathrm{year}$ comes just from cooperation and trade, while the best target type adds another $\$ 17.6$ bn/year.

\subsection{Targets and optimal indexation by country}

The globally averaged emissions target is, respectively, 2.8 and 3.2 percentage points more stringent under standard and optimal intensity targets than under absolute targets (Table 3). For individual countries, the changes in endogenous targets vary greatly, here ranging from less than one to around ten percentage points. ${ }^{16}$ This is because the degree to which intensity targets can help address uncertainty varies across countries. Neutralising activity-related emissions uncertainty brings the greatest advantage where the share of emissions linked with GDP $\left(\alpha_{i}\right)$ is large, where uncertainty about future GDP $\left(\sigma_{Y i}\right)$ is large relative to other uncertainties, and where risk aversion $\left(z_{i}=1 / y_{i}\right)$ is strong. In addition, larger countries tend to be affected more strongly by emissions uncertainty (and benefit from reducing uncertainty), as their domestic circumstances affect the global permit price more.

For Southern (mainly developing) countries as a group, the impact of indexation on optimal target levels is greater than for Northern countries. In particular, moving from standard to optimal intensity targets brings little change for North's targets overall, but rather more stringent targets in many Southern countries. So customising the degree of indexation plays a more important role in developing than in industrialized countries. This is because of systematic differences in the degree of emissions-GDP linkage $\alpha_{i}$ and relative targets $x_{i}$ between countries, which enter the optimality condition $\beta_{i}^{*}=\alpha_{i} / x_{i}$ in (2.9). In many industrialized countries $\alpha_{i}$ takes on comparatively large values, because of the dominance of emissions from fossil fuel combustion (Australia and Canada/New Zealand being notable exceptions). At the same time, richer countries get allocated comparatively stringent targets, so $x_{i}$ are low. Together, this gives $\beta_{i}^{*}$ in the broad vicinity of one for many 'Northern' countries, so standard intensity targets with one-to-one indexation would already do quite well.

For several countries, the constrained-optimal target level is lower than the share of emissions linked with GDP, so super-indexation of targets is optimal $\left(x_{i}<\alpha_{i}\right.$ means $\left.\beta_{i}^{*}>1\right)$. Under a reasonably ambitious treaty, this is likely to happen in many advanced economies, with Japan's super-indexation of 1.3 being highest in our scenario.

In most developing countries by contrast, partial indexation would be optimal. Countries with large non-energy sector emissions would generally be best off with very low degrees of indexation. This is evident for Indonesia and Brazil, where a large share of emissions stems from deforestation, the rate of which is unlikely to move with

\footnotetext{
16 The move to a more flexible target type can lead to slightly less stringent targets (higher $x_{i}$ ) in some countries/regions, contrary to more stringent commitments in aggregate. This is because reducing the cost of uncertainty has disproportionately large effects in some countries, and these gains are distributed across all countries under our equity rule (3.16), by way of adjusting target levels.
} 
Table 3 Intensity targets by country

\begin{tabular}{|c|c|c|c|c|c|c|}
\hline \multirow{3}{*}{$\begin{array}{l}\text { Country/region } \\
(\mathrm{N}=\text { North })\end{array}$} & \multirow{2}{*}{\multicolumn{2}{|c|}{$\begin{array}{l}\text { Reference case } \\
\text { Absolute targets } \\
\left(\beta_{i}=0\right)\end{array}$}} & \multicolumn{3}{|c|}{ Endogenous intensity targets } & \multirow{2}{*}{$\begin{array}{l}\text { Share of } \\
\text { emissions } \\
\text { linked } \\
\text { with GDP }\end{array}$} \\
\hline & & & $\begin{array}{l}\text { Standard } \\
\text { intensity } \\
\text { targets } \\
\left(\beta_{i}=1\right)\end{array}$ & $\begin{array}{l}\text { Optimal } \\
\text { intensity } \\
\text { targets } \\
\left(\beta_{i}^{*}=\alpha_{i} /\right. \\
\left.x_{i}\right)\end{array}$ & $\begin{array}{l}\text { Index- } \\
\text { ation } \\
\text { under } \\
\text { optimal } \\
\text { targets }\end{array}$ & \\
\hline & $\begin{array}{l}\text { Abated } \\
\text { emis. } \\
\text { relative } \\
\text { to BAU } \\
\text { emis. } \\
\left(E_{i} / E_{i}^{b}\right)\end{array}$ & $\begin{array}{l}\text { Target } \\
\text { relative to } \\
\text { BAU } \\
\text { emis. }\left(x_{i}\right)\end{array}$ & \multicolumn{2}{|c|}{$\begin{array}{l}\text { Target relative to BAU } \\
\text { emissions }\left(x_{i}\right)\end{array}$} & $\beta_{i}^{*}$ & $\alpha_{i}$ \\
\hline United States $(\mathrm{N})$ & 0.913 & 0.812 & 0.781 & 0.780 & 1.06 & 0.83 \\
\hline Europe $(\mathrm{N})$ & 0.933 & 0.748 & 0.727 & 0.727 & 1.13 & 0.82 \\
\hline Japan (N) & 0.971 & 0.750 & 0.739 & 0.739 & 1.30 & 0.96 \\
\hline Australia (N) & 0.874 & 0.810 & 0.796 & 0.795 & 0.81 & 0.65 \\
\hline Canada/NZ (N) & 0.915 & 0.823 & 0.811 & 0.811 & 0.80 & 0.65 \\
\hline Russia & 0.836 & 0.801 & 0.773 & 0.773 & 1.01 & 0.78 \\
\hline China & 0.829 & 0.957 & 0.853 & 0.852 & 0.92 & 0.78 \\
\hline India & 0.853 & 0.947 & 0.936 & 0.936 & 0.65 & 0.61 \\
\hline Brazil & 0.876 & 0.904 & $0.897^{\mathrm{a}}$ & 0.891 & 0.25 & 0.22 \\
\hline Argentina & 0.890 & 0.853 & $0.843^{\mathrm{a}}$ & 0.843 & 0.45 & 0.38 \\
\hline Mexico & 0.884 & 0.832 & 0.819 & 0.819 & 0.79 & 0.65 \\
\hline Korea (S.) & 0.923 & 0.815 & 0.801 & 0.800 & 1.16 & 0.93 \\
\hline Indonesia & 0.859 & 0.928 & $0.922^{\mathrm{a}}$ & 0.914 & 0.21 & 0.19 \\
\hline South-East Asia & 0.876 & 0.899 & $0.891^{\mathrm{a}}$ & 0.888 & 0.47 & 0.42 \\
\hline South Africa & 0.861 & 0.810 & 0.796 & 0.795 & 1.12 & 0.89 \\
\hline Northern Africa & 0.892 & 0.916 & 0.908 & 0.908 & 0.79 & 0.72 \\
\hline Middle East & 0.875 & 0.914 & 0.895 & 0.895 & 0.79 & 0.71 \\
\hline $\begin{array}{l}\text { Rest of world } \\
\text { A goregates. }\end{array}$ & 0.882 & 1.014 & $1.026^{\mathrm{a}}$ & 1.004 & 0.34 & 0.34 \\
\hline $\begin{array}{l}\text { Aggregates: } \\
\text { North }\end{array}$ & & & & & & \\
\hline North & 0.923 & 0.785 & 0.761 & 0.761 & - & 0.82 \\
\hline South & 0.860 & 0.929 & 0.899 & 0.893 & - & 0.55 \\
\hline Global & 0.881 & 0.881 & 0.853 & 0.849 & - & 0.64 \\
\hline
\end{tabular}

${ }^{\text {a }}$ For these countries, the standard intensity target $x_{i}$ exceeds $2 \alpha_{i}$, and so reduces expected payoff compared to the Reference Case

overall GDP. Here, optimal indexation would be very low for a treaty that includes all greenhouse gas sources. Determining the optimal degree of indexation for each country in practice would require empirical assessments of emissions-GDP linkage at the sub-sectoral level, and the sectoral coverage of an emissions target would be an important determinant of optimal indexation.

In some countries, $x_{i}>2 \alpha_{i}$, so from (2.12) overcompensation under standard intensity targets would actually increase emissions uncertainty. If continuously differentiated indexation were not an option and each country only had the dichotomous choice between an absolute or a standard intensity target $\left(\beta_{i}=0\right.$ or 1$)$, then global payoff would be maximized if some developing countries had absolute, and the rest had standard intensity targets. In our scenario, absolute targets are preferable for Brazil, Indonesia, the Rest of the World region (all of which have large emissions 
from land-use change) as well as - only just-Argentina and the South-East Asia region. ${ }^{17}$ Optimally indexed targets would of course be preferable in all countries.

\subsection{Sensitivity analysis}

Here we test the sensitivity of results to changes in some key parameters and assumptions from the baseline values used so far (as described in Sect. 3.4 and the Appendix), for the scenarios with endogenous intensity targets. Results are given in Table 4, with comments as follows.

\subsubsection{Equity criteria}

As foreshadowed earlier, our choice of equal per capita payoff as our standard equity criterion in (3.16) affects target levels and differentiation between countries, but makes little difference to the relative assessment of different types. For example, if the average global emissions reduction were applied uniformly to all countries (i.e. $x_{i}=0.88$ for all countries in the Reference Case, and likewise uniform percentage reductions under endogenous intensity targets), this would leave Southern countries with a much greater, and Northern countries with a much lesser, burden than in our Reference Case. Yet the increases in (global expected) gain, payoff and abatement from intensity targets differ only moderately from our baseline results.

\subsubsection{Uncertainty about GDP}

In further sensitivity analysis, the GDP uncertainty parameter $\sigma_{Y i}$ is either reduced or increased by one-third, compared to the baseline calibration. Predictably, the less reliable projections of future GDP are, the greater is the potential role for intensity targets. Also, the gap between standard and optimal indexation increases in $\sigma_{Y i}$. Greater uncertainty about future GDP thus not only makes intensity targets more attractive, but also makes optimal indexation relatively more attractive, an outcome also found by Sue Wing et al. (forthcoming) for the single-region case.

\subsubsection{Risk aversion}

If risk aversion is stronger than in our baseline calibration, the advantage of intensity targets is also greater; while if parties are less averse to the risk of losses, then designing mechanisms to mitigate risk is less important also. The greater the degree of risk aversion, the greater the increase in expected global abatement under endogenous targets - ranging from no or almost no change under risk neutrality, to much larger improvements than in our baseline results. Gain $G$ and payoff $U$ however are greater with intensity targets even under risk neutrality, as they are a function of net uncertainty at any given level of abatement.

17 By contrast, Sue Wing et al. (forthcoming) found intensity targets preferable to absolute targets for all six developing countries they looked at. This is probably because they considered data for $\mathrm{CO}_{2}$ from fossil fuel combustion only, whereas we include all major greenhouse gas emissions and sources. 
Table 4 Sensitivity analysis

\begin{tabular}{|c|c|c|c|}
\hline \multirow[t]{2}{*}{ Scenario } & \multirow[t]{2}{*}{$\begin{array}{l}\text { Change to parameters } \\
\text { (applies to all countries) }\end{array}$} & \multicolumn{2}{|c|}{$\begin{array}{l}\text { Global change compared to absolute targets, } \\
\text { under endogenous optimal intensity targets }\end{array}$} \\
\hline & & $\begin{array}{l}\text { Expected } \\
\text { Abatement } Q(\%)\end{array}$ & $\begin{array}{l}\text { Expected Gain } \\
G(\%)\end{array}$ \\
\hline Baseline & - & 27 & 17 \\
\hline $\begin{array}{l}\text { Uniform reduction } \\
\text { commitments }\end{array}$ & Same $x_{i}$ for all countries & 21 & 16 \\
\hline Low GDP uncertainty & $\sigma_{Y i}$ reduced by one-third & 12 & 6 \\
\hline High GDP uncertainty & $\begin{array}{l}\sigma_{Y i} \text { increased by } \\
\text { one-third }\end{array}$ & 41 & 34 \\
\hline No risk aversion & $r=0$ & 0 & 6 \\
\hline High risk aversion & $\begin{array}{l}r=0.1 \text { (baseline } \\
r=0.085)\end{array}$ & 37 & 26 \\
\hline $\begin{array}{l}\text { Weaker GDP-emis- } \\
\text { sions link }\end{array}$ & $\alpha_{i}$ halved & 4 & 2 \\
\hline $\begin{array}{l}\text { Stronger GDP-emis- } \\
\text { sions link }\end{array}$ & $\left(1-\alpha_{i}\right)$ halved & 36 & 27 \\
\hline
\end{tabular}

\subsubsection{GDP-emissions linkage}

We first assume that only half (instead of all, as in the baseline) of energy sector emissions are linked with GDP, resulting in lower values for $\alpha_{i}$ across the board. Standard intensity targets on the whole then perform slightly worse than absolute targets, because they strongly overcompensate for fluctuations in GDP. Optimal intensity targets, at low levels of indexation, still improve the outcome compared to absolute targets. If instead the overall emissions-GDP linkage is stronger than in the baseline, both standard and optimal intensity targets bring greater improvements over absolute targets.

\section{Drawbacks of target indexation}

Here we discuss the key downsides of intensity targets, and address arguments made against indexation in some of the relevant policy literature. The discussion here goes beyond what is captured in our formal model.

\subsection{Framing of uncertainty}

In the model we have assumed that the (risk-adjusted) payoff a country perceives from joining an emissions trading treaty is framed as a function of just the financial and environmental consequences of joining the treaty, not of any broader economic stabilisation. This would seem logical from the point of view of the treaty negotiators and industries that will be subject to emissions control, but from the point of the view of the country, should not the broader effect of overall economic uncertainty on welfare be taken into account?

Which frame of reference to use for negotiation is a matter for political and psychological choice. Given the existence of emissions trading, an absolute emissions target is not an absolute constraint on GDP growth, but a financial stabiliser. Intensity targets 
by contrast are pro-cyclical. However, our reading of the debate is that governments and negotiators are much more concerned about uncertainty within a treaty and their obligations under it. Potential impacts on domestic industries' competitiveness have played a strong role in the Kyoto Protocol negotiations, but arguments of overall economic stabilization were rarely heard. The value of permits would be small relative to the size of economies (in our calibration around $0.8 \%$ of global GDP), so changes in permit allocations would only amount to very small fractions of the changes in GDP that trigger them.

\subsection{Shifting uncertainty to emission levels}

Under intensity targets, the overall amount of emissions allowable under a treaty with intensity targets is not fixed, so uncertainty to some degree is shifted away from costs and on to emissions levels (Dudek and Golub 2003). We find that this is not an effective argument against target indexation in the greenhouse case, for several reasons.

First, we have shown that intensity targets can achieve both more stringent environmental commitments in expectation terms, and higher welfare. So higher variability in $\widetilde{X}$, the amount of realized emissions allowed under the treaty, is outweighed in welfare terms by a lower level of $X$, the expected emissions. Under our simulation scenarios, there is a greater than $80 \%$ chance that global emissions $\widetilde{X}$ under the treaty with optimal intensity targets are lower than under absolute targets, and actual abatement undertaken $\left(\widetilde{Q}=\widetilde{E}^{b}-\widetilde{X}\right)$ is also greater in the majority of random realisations. And while intensity targets raise variability in total emissions, they actually lower variability in the amount of abatement.

Second, uncertainty about overall emissions is created only when fluctuations do not cancel out between countries, that is under a global economic boom or slowdown. A more typical pattern is for some countries to grow slower than expected while others grow faster, with offsetting effects.

Finally, variability in emissions as a result of indexation of medium-term targets has a negligible effect on the existing, long-lived stock of greenhouse gases in the atmosphere. For example, the global atmospheric concentration of carbon dioxide is currently growing by about $0.4 \%$ per year, and fluctuations in emissions because of target indexation will amount to only a small share of this $0.4 \%$. So any temporary over- or undershooting of desired emissions at some point in time can be compensated for by periodically re-negotiating expected target levels.

\subsection{Complexity and window-dressing}

By bringing into play GDP as an additional variable for permit allocation, intensity targets make monitoring, verification and administration of the trading scheme more complicated, and there may be complications with using GDP measures as an activity index, especially with regard to inflation-proofing (Müller and Müller-Fürstenberger 2003). Intensity targets would thus add extra administrative complexity to the challenges, such as distributional impacts and the risk of Dutch disease from selling permits, that any kind of emissions trading already poses for developing countries. Further, differentiating the degree of indexation would pose extra challenges for negotiations. 
There is also a danger that the very framing of targets in terms of intensity could be used to undermine the environmental stringency of commitments at a political level, in a highly charged and often poorly informed public debate. A reduction in future greenhouse intensity looks more stringent than its equivalent expressed as a change in absolute emissions, because emissions intensity in most countries declines over time anyway, and this can be used for political window-dressing. For example, the Bush administration announced a goal of an $18 \%$ decline in US greenhouse emissions intensity from 2002 to 2012. This is close to the expected business-as-usual path, and implies a substantial increase in emission levels, despite it being framed as a 'reduction' (in intensity). Window-dressing could be dealt with by presenting intensity targets in terms of their expected absolute emission levels with an adjustment term, as in (2.3).

\section{Conclusions}

Uncertainty about future paths of economic and emissions growth can be an important obstacle to effective cap-and-trade schemes for emissions control, because of ex ante uncertainty about the cost of complying with emissions targets. Intensity targets, where permit allocations are linked to future uncertain GDP, have been proposed as a means to reduce cost uncertainty by compensating for activity-related fluctuations in emissions. We have taken a theoretical model of an emissions trading treaty with endogenous intensity targets under GDP-linked and other uncertainties in emissions, applied it in a new multi-country, single-period, mainly partial equilibrium, empirical simulation model of global, greenhouse emissions trading under uncertainty with risk aversion, and discussed the findings in a policy context. Target levels were set endogenously by minimising a fairly egalitarian definition of global welfare, but we found that using another definition would make very little difference to our comparisons across different target types.

We have shown how, under which conditions, and by how much intensity targets could improve both participating countries' welfare and the environmental stringency of a 2020 cooperative climate treaty that covers all countries and the majority of greenhouse gases. Standard intensity targets, which are indexed one-to-one to GDP, can reduce overall cost uncertainty and lead to better outcomes than Kyoto-Protocol-style absolute targets, but they can systematically over- or undercompensate for GDPrelated fluctuations. Optimal intensity targets, where the degree of indexation is the ratio of the extent of each country's GDP-emissions linkage to its proportional target commitment, always perform better than absolute targets, because they always neutralise some degree of risk aversion. In the scenarios simulated here, optimal intensity targets would result in increases in expected global abatement of more than a quarter, and in expected global welfare of more than a third, above the cooperation and trade benefits already achievable by absolute targets. The quantitative results for endogenous targets depend on assumptions about risk aversion, while there are underlying welfare gains even with fixed targets and zero risk aversion. The quantitative results also may change with improved empirical estimates of the magnitude of uncertainties, and with assumptions on their correlations.

In our model we find that optimal degrees of indexation would differ strongly between countries, with 'super-indexation' optimal for many rich countries. For example, we find that Japan's and Europe's emissions targets would optimally rise or fall by about 1.3 and $1.1 \%$, respectively, if their GDPs turn out $1 \%$ higher or lower than 
expected. For developing countries by contrast, optimal indexation would typically be only partial, for targets that cover all greenhouse gas emissions. This makes it all the more important to tailor indexation to countries' circumstances. In five out of our 18 regions, an absolute target would actually be preferable to a standard intensity target if a discrete choice had to be made, though an optimal intensity target always dominates.

Intensity targets have downsides, including their pro-cyclical effects in terms of the economy as a whole, their greater complexity and lesser transparency, and a small uncertainty about the level of emissions under the treaty. None of these potential drawbacks are likely to deal intensity targets a fatal blow however, and they need to be assessed against the important opportunity highlighted here, that greater flexibility could both strengthen environmental commitments and raise welfare. A more complex, dynamic general equilibrium analysis of intensity targets seems unlikely to change this key conclusion.

In the context of ongoing UN climate negotiations, intensity targets may be particularly interesting for middle-income, industrializing countries that consider joining a successor treaty to the Kyoto-Protocol. Further down the track they might be attractive for some developing countries. They could also help make stringent national-level emissions targets politically more palatable in developed countries, perhaps even the US. Target indexation is unlikely to be sufficient by itself, though, to overcome deepseated problems of cooperation, equity and politics that lie at the heart of the deadlock in global climate negotiations. Economic restructuring to achieve deep cuts in global greenhouse emissions will be costly, and rich countries will have to pay if it is to happen. Yet although flexible targets are no magic wand, in some cases they might just tip the political balance in favour of making a meaningful climate treaty work.

\section{Appendix: Calibration of the MATES model for greenhouse gases in 2020}

\subsection{Emissions, GDP and population}

Data for the base year 2000 are from the World Resource Institute's CAIT database Version 3.0 (WRI 2005), which compiles data from a range of sources. In calibrating BAU emissions $E_{i}^{b}$ we include carbon dioxide $\left(\mathrm{CO}_{2}\right)$ from the energy sector (combustion of fossil fuels, plus emissions from cement production), $\mathrm{CO}_{2}$ from land-use change (mainly deforestation in tropical countries), and emissions of methane and nitrous oxide from a range of sources. Base data are for $2002\left(\mathrm{CO}_{2}\right)$ and 2000 (other

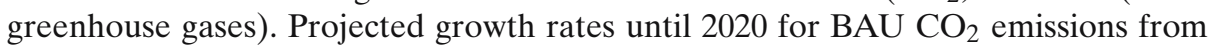
the energy sector are taken from projections by the US Energy Information Administration (EIA 2004) and International Energy Agency (IEA 2004). For non-energy GHG emissions, we use projections from US-EPA (2006). For land-use change we assume that annual emissions remain constant, in the absence of reliable projections. For GDP, we use purchasing power parity (PPP-) adjusted GDP in the year 2002 as the base data, and projections from EIA and IEA. Population growth is from United Nations (2004) projections. Base data again are from CAIT. All the resulting projections for 2020 are in Table 5. 


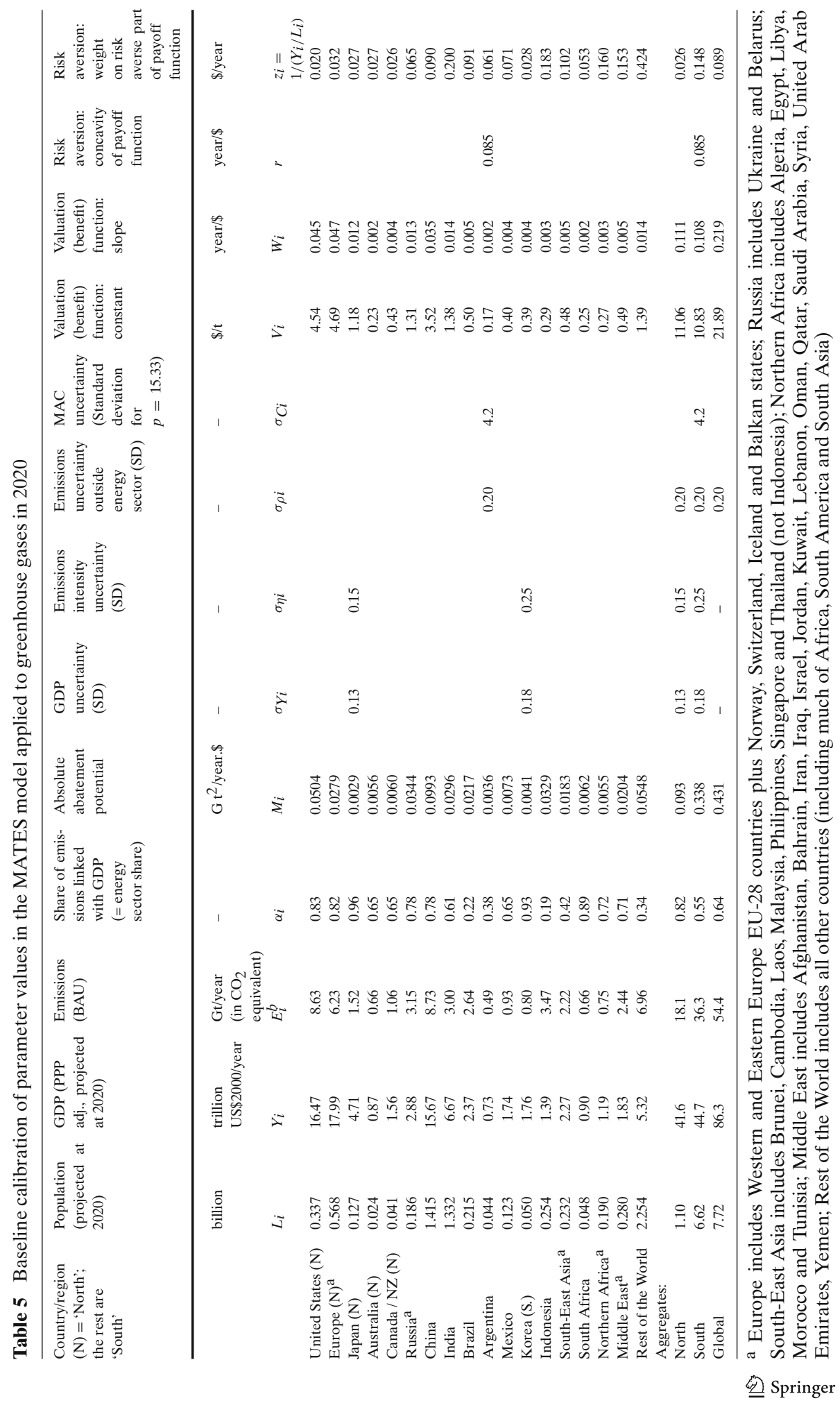




\subsection{Marginal abatement cost (MAC) curves}

Abatement potentials $M_{i}$ are calibrated for energy-derived $\mathrm{CO}_{2}$ on the basis of structural characteristics of each country. The relationship between abatement potential, emissions intensity of electricity production, and the overall emissions intensity of the economy, is estimated using abatement cost estimates from computable general equilibrium models (Ellerman and Decaux 1998; Polidano et al. 2000). This yields a consistent set of abatement potentials for our calibration, which is more regionally disaggregated than published MAC estimates (see also Jotzo 2006a). For non- $\mathrm{CO}_{2}$ emissions we derive relative abatement parameters from data cited by den Elzen and Lucas (2004); for land-use change, we assume that relative abatement potential is broadly in line with that for non- $\mathrm{CO}_{2}$ emissions. MAC schedules in the model are linear. Linearisation does not greatly change estimates of total costs compared to empirically estimated MAC functions with a typical degree of convexity, for the levels of abatement considered in this application.

\subsection{Uncertainties and the GDP-emissions link}

The empirical basis for calibrating uncertainty parameters $\sigma_{Y i}, \sigma_{\eta i}$ and $\sigma_{\rho i}$ and the degree of GDP-emissions linkage $\alpha_{i}$ is described in Sect. 2.1, and further detail can be found in Jotzo (2006b). The abatement cost uncertainty parameter $\sigma_{C i}$ was estimated on the basis of the divergence between estimates for marginal abatement costs from ten models (Weyant 1999), and calibrated for $p=15 \$ / t$ as in our Reference Case.

All uncertainty parameters $\sigma$ are scaled back by one third compared to the original empirical estimates, in order to reduce any risk of overstating the degree of effective uncertainty in our model. The distribution of error terms $\varepsilon$ is truncated at two standard deviations above and below zero, in order to exclude unrealistic realizations at the extreme tails of the probability distributions. Errors are thus truncated in just under $5 \%$ of individual realisations.

The parameter $\alpha_{i}$ (emissions linked with GDP) is set equal to the share of emissions from fossil fuel combustion and cement manufacturing in total emissions, projected for the year 2020 .

\subsection{Valuation of emissions reductions}

\section{See Section 3.4.}

Acknowledgements We thank Ken Arrow, Larry Goulder and David Victor for expert guidance of the project of which this paper is one output. For funding we thank the William and Flora Hewlett Foundation through Stanford University, as well as the Program on Energy and Sustainable Development at Stanford University. We are grateful to Quentin Grafton and Jason Sharples for valuable advice, and for helpful comments we thank Paul Baer, Kevin Baumert, Tony Beck, Michel den Elzen, Charlie Kolstad, Warwick McKibbin, Klaus Oppermann, Cédric Philibert, Rob Williams and seminar participants at the 2004 AARES annual meetings, the 2004 EAERE conference, the 2005 EEN workshop, at Australian National University, Hamburg Institute, ZEW Mannheim, Stanford University, UC Berkeley and UC Santa Barbara. Two anonymous referees gave many valuable comments. Any errors are our own. 


\section{References}

Asafu-Adjaye J (2000) The relationship between energy consumption, energy prices and economic growth: time series evidence from Asian developing countries. Energy Econ 22(6):615-625

Barros V, Conte Grand M (2002) Implications of a dynamic target of greenhouse gases emission reduction: the case of Argentina. Environ Dev Econ 7(3):547-569

Baumert KA, Bhandari R, Kete N (1999) What might a developing country climate commitment look like? World Resources Institute, Washington, DC

Berk MM, den Elzen MGJ (2001) Options for differentiation of future commitments in climate policy: how to realise timely participation to meet stringent climate goals? Clim Policy 1:465-480

Blanchard O, Perkaus JF (2004) Does the Bush Administration's climate policy mean climate protection? Energy Policy 32(18):1993-1998

Bohm P, Carlén B (2002) A cost-effective approach to attracting low-income countries to international emissions trading: theory and experiments. Environ Resour Econ 23:187-211

Bouille D, Girardin O (2002) Learning from the Argentine voluntary commitment. In: Baumert KA, Blanchard O, Llosa S, Perkaus JF (eds) Building on the Kyoto Protocol: Options for protecting the climate. World Resources Institute, Washington DC

den Elzen MGJ, Lucas P (2004) FAIR 2.0 - A decision-support tool to assess the environmental and economic consequences of future climate regimes. RIVM report 550015001/2003, RIVM, Bilthoven

Dinda S (2004) Environmental Kuznets Curve hypothesis: a survey. Ecol Econ 49(4):431-455

Dudek D, Golub A (2003) "Intensity" targets: pathway or roadblock to preventing climate change while enhancing economic growth? Climate Policy 3(Suppl. 2):S21-S28

Eckersley R (2004) Soft Law, Hard Politics and the Climate Change Treaty. In: Reus-Smit C (ed) The Politics of International Law. Cambridge University Press, Cambridge

EIA (2004) International Energy Outlook 2004. US Energy Information Administration, Washington, DC

Ellerman AD, Decaux A (1998) Analysis of Post-Kyoto CO2 emissions trading Using Marginal Abatement Curves, MIT Joint Program on the Science and Policy of Global Change, Report no. 40, Cambridge, MA

Ellerman AD, Sue Wing I (2003) Absolute versus intensity-based emission caps. Climate Policy 3(Suppl. 2):S7-S20

Frankel JA (1999) Growth Baselines: reducing emissions and increasing investment in developing Countries. Brookings Policy Brief no. 52, Brookings Institution, Washington, DC

Galeotti M, Lanza A, Pauli F (2006) Reassessing the environmental Kuznets curve for CO2 emissions: a robustness exercise. Ecol Econ 57(1):152-163

Höhne N, Harnisch J (2002) Greenhouse gas intensity targets vs. absolute emissions targets. Paper presented at the Sixth International Conference on Greenhouse Gas Control Technologies. ECOFYS, Cologne

Holtz-Eakin D, Selden TM (1995) Stoking the fires? CO2 emissions and economic growth. J. Public Econ 57(1):85-101

IEA (2004) World Energy Outlook 2004. OECD/International Energy Agency, Paris

Jotzo F (2006a) Global climate policy after the Kyoto Protocol: flexible economic mechanisms for South and North under uncertainty and institutional constraints. Ph.D thesis, Australian National University, Canberra

Jotzo F (2006b) Quantifying uncertainties for emissions targets. Economics and Environment Network Working Paper EEN0603. Australian National University, Canberra

Kim Y-G, Baumert KA (2002) Reducing uncertainty through dual-intensity targets. In: Baumert KA, Blanchard O, Llosa S, Perkaus JF (eds) Building on the Kyoto Protocol: Options for protecting the climate. World Resources Institute, Washington DC

Kolstad CD (2005) The simple analytics of greenhouse gas emission intensity reduction targets. Energy Policy 33(17):2231-2236

Lutter R (2000) Developing countries greenhouse emissions: uncertainty and implications for participation in the Kyoto Protocol. Energy J. 21(4):93-120

Müller B, Müller-Fürstenberger G (2003) Price-related sensitivities of greenhouse gas intensity targets. Climate Policy 3(Suppl 2):S59-S74

Nordhaus WD (1991) To slow or not to slow: the economics of the greenhouse effect. Econ J 101(407):920-937

Oh W, Lee K (2004) Causal relationship between energy consumption and GDP revisited: the case of Korea 1970-1999. Energy Econ 26(1):51-59 
Pezzey J, Jotzo F (2007) Mechanisms for abating total emissions under uncertainty. Economics and Environment Network Working Paper EEN0702, Australian National University, Canberra. At http://een.anu.edu.au/papers

Philibert C (2004) Flexible targets for developing countries. Paper presented at the 'International seminar on climate change', Seoul

Philibert C, Pershing J (2001) Considering the options: climate targets for all countries. Climate Policy 1(2):211-227

Pizer WA (2002) Combining price and quantity controls to mitigate global climate change. J Public Econ 85:409-434

Polidano C, Jotzo F, Heyhoe E, Jakeman G, Woffenden K, Fisher BS (2000) The Kyoto Protocol and Developing Countries: Impacts and Implications for Mechanism Design. ABARE Research Report 2000.4, Australian Bureau of Agricultural and Resource Economics, Canberra

Quirion P (2004) Prices versus Quantities in a Second-Best Setting. Environ Resour Econ 29(3):337360

Quirion P (2005) Does uncertainty justify intensity emission caps? Resour Energy Econ 27(4):343-353

Rehdanz K, Tol RSJ (2005) Unilateral regulation of bilateral trade in greenhouse gas emission permits. Ecol Econ 54(4):397

Rose A, Stevens B, Edmonds J, Wise M (1998) International equity and differentiation in global warming policy: an application to tradeable emission permits. Environ Resour Econ 12(1):25-51

Schmalensee R, Stoker TM, Judson RA (1998) World Carbon Dioxide Emissions: 1950-2050. Rev Econ Stat 80(1):15-27

Stavins RN (1996) Correlated Uncertainty and Policy Instrument Choice. J Environ Econ Manage 30:218-232

Sue Wing I, Ellerman AD, Song J (forthcoming) Absolute vs. intensity limits for $\mathrm{CO} 2$ emissions control: performance under uncertainty. In: Tulkens H, Guesnerie R (eds) The design of climate policy. MIT, Cambridge, MA

Tol RSJ (2005) The marginal damage costs of carbon dioxide emissions: an assessment of the uncertainties. Energy Policy 33(16):2064-2074

Toman MA (2004) Economic analysis and the formulation of U.S. Climate Policy. In: Lutter R, Shogren JF(eds) Painting the White House Green: Rationalizing Environmental Policy Inside the Executive Office of the President. Resources for the Future, Washington, DC

United Nations (2004) World population prospects: the 2004 revision and World urbanization prospects. Population Division of the Department of Economic and Social Affairs of the United Nations Secretariat, New York

US EPA (2006) Global Mitigation of Non-CO2 Greenhouse Gases. United States Environmental Protection Agency, Office of Atmospheric Programs, Washington, DC

van Vuuren D, den Elzen M, Berk M, de Moor A (2002) An evaluation of the level of ambition and implications of the Bush Climate Change Initiative. Climate Policy 2(4):293-301

Webster M, Cho C-H (2006) Analysis of variability and correlation in long-term economic growth rates. Energy Econ 28(5-6):653-666

Weitzman ML (1974) Prices vs Quantities. Rev Econ Stud 41(4):477-491

Weyant J (1999) The costs of the Kyoto Protocol: a multi-model evaluation. Energy J (Special Issue)

WRI (2005) Climate analysis indicator tool. version 3.0, World Resources Institute, Washington, DC. http://cait.wri.org 